\title{
FINITE SOLUBLE GROUPS WITH CERTAIN SPLITTING PROPERTIES
}

\author{
R. A. BRYCE and JOHN COSSEY
}

(Received 18 May 1971)

Communicated by G. E. Wall

Abstract. In a recent paper, Bechtell obtained detailed structure theorems for finite supersoluble groups with the property that every minimal supplement for a non-Frattini normal subgroup is a complement. We consider finite soluble groups with this property. The situation is rather different to the supersoluble case, and the information we obtain is not as complete, though for such groups with non-trivial Frattini subgroup, some of the results are analogous.

\section{Introduction}

In a recent paper, Bechtell [1] studies finite supersoluble groups $G$ with the properties:

$\mathscr{P}$ : if $N$ is a normal subgroup of $G$ not in the Frattini subgroup $\Phi G$ of $G$, then $N$ is complemented in $G$,

$\mathscr{P}^{*}:$ if $N$ is a normal subgroup of $G$ not in $\Phi G$, then every minimal supplement for $N$ in $G$ is a complement.

He finds detailed structure theorems for such groups, the interesting case for supersoluble groups being groups with non-trivial Frattini subgroups. In the present paper, we shall be mainly concerned with finite soluble $\mathscr{P} *$ groups-not necessarily supersoluble ones (and from now on all groups will be assumed to be finite and soluble). The situation here is quite different to the supersoluble case, and few of Bechtell's results go over, though there are some analogues for the case of non-trivial Frattini subgroup.

A well known result of Gaschütz ([5] Satz 8) is that in a group with elementary abelian Sylow subgroups every normal subgroup has a complement: it follows easily that such a group is a $\mathscr{P} *$ group. The converse of this theorem is false: witness $S_{4}$. However, $S_{4}$ is not a $\mathscr{P *}$ group, and one is tempted to conjecture that being $\mathscr{P}^{*}$ and $\Phi$-free ensures elementary abelian Sylow subgroups. We shall show that this is indeed so for groups of nilpotent length three, and give an example (of nilpotent length four) to show that it is not true in general. Side by side with this example, we construct another group which is not a $\mathscr{P} *$ group, but is similar enough in structure to make it seem unlikely that an easily applicable structure theorem can 
be found which will be fine enough to distinguish between these two groups. We can obtain a structure theorem which places some limitations on the stucture of certain Sylow subgroups, and it turns out to be quite useful.

It is known (Christensen [3]) that the class of $\Phi$-free $\mathscr{P}$ groups is a formation. The same is not true for the class of $\Phi$-free $\mathscr{P} *$ groups, and we show that this class is not even direct product closed. One might then ask whether the formation generated by a $\Phi$-free $\mathscr{P}^{*}$ group consists of $\mathscr{P}^{*}$ groups: the answer is again no but it is somewhat harder to settle: the example is fairly complicated, and we shall not write it down. We shall prove however that if $H$ has elementary abelian Sylow subgroups, and $G$ is a $\Phi$-free $\mathscr{P} *$ group with elementary abelian Sylow $p$-subgroups for those primes $p$ dividing the order of $H$, then $G \times H$ is a $\mathscr{P}^{*}$ group.

Finally, we consider $\mathscr{P}^{*}$ groups with non-trivial Frattini subgroup. Here we can obtain a certain amount of information on the Fitting subgroup, the most general result being for a $\mathscr{P}^{*}$ group $G$, with $\Phi G \neq 1, F(G) / \Phi G$ has at most two irreducible components.

\section{Notation and Preliminaries}

For a group $G, \Phi G$ denotes the Frattini subgroup of $G$, and $F(G)$ the Fitting subgroup of $G$. If $\Phi G=1$, we say $G$ is $\Phi$-free. We shall use, often without comment, a number of facts about the Frattini and Fitting subgroups and the relation between them, and so we assume familiarity with the results of Gaschütz [6]. Notation is usually standard, or explained as it is introduced.

If $\pi$ is a set of primes, then for a group $G, G_{\pi}$ will denote a Hall $\pi$-subgroup of $G$, and $\mathscr{E}_{\pi}$ the class of $\pi$-groups with elementary abelian Sylow subgroups. We shall need the following result:

2.1 If $G$ is a group with $G_{\pi} \in \mathscr{E}_{\pi}$, and $N$ is a normal $\pi$-subgroup of $G$, then $N$ is complemented in $G$.

This result is easily deduced from Gaschütz [5] Reduktionssatz 2.

We shall need several elementary facts about $\mathscr{P}$ groups and $\mathscr{P} *$ groups, and we list them (without proof) below. The proofs of those for which no reference is given are easy.

2.2 Each homomorphic image of a group $G \in \mathscr{P}($ or $\mathscr{P} *)$ has property $\mathscr{P}$ $\left(\right.$ or $\left.\mathscr{P}^{*}\right)$.

2.3 If $G \in \mathscr{P}$, and $N$ is normal subgroup of $G$, then either $N \leqq \Phi G$, or $\Phi G<N$.

2.4 If $G \in \mathscr{P}$, and $\Phi G \neq 1, F(G)$ is a p-group for some prime $p$.

2.5 If $G \in \mathscr{P}$, and $Z(G)$ is the centre of $G$, then 
(i) $Z(G) \leqq \Phi G$, or

(ii) $G=H \times Z(G)$, where $Z(H)=\Phi H=1$.

2.6 If $G \in \mathscr{P}$, then either $Z(F(G)) \leqq \Phi G$ or $F(G)$ is abelian.

2.7 If $G \in \mathscr{P}, \Phi G=1$, and $N$ is a normal subgroup of $G$, then $\Phi(G / N)=1$.

2.8 (Christensen [2] Theorem 3.5) If $G \in \mathscr{P}, \Phi G=1$, and $N$ is a normal subgroup of $G$, then $N \in \mathscr{P}$.

It is often convenient to regard chief factors and elementary abelian normal subgroups of a group as modules for the group: we shall do so without comment whenever it seems convenient.

\section{Two examples}

In this section we construct two very similiar groups, one of which is a $\mathscr{P} *$ group, the other not.

Both groups have a common start to their construction. We begin with $S_{3}$, which we take to be generated by elements $a, b$ with $a^{2}=b^{3}=1 . S_{3}$ has a faithful irreducible representation of degree two over $G F(7)$ : let $M$ be a module affording this representation. The following facts about $M$ are easily deduced: $M$ is induced from a one-dimensional faithful module for $\langle b\rangle$ over $G F(7)$ (use e.g. Blichfeldt's Theorem [4] 50.7), and $M_{\langle a\rangle}$ is the regular representation of $\langle a\rangle$ (use e.g. the Mackey Subgroup Theorem [4] 44.2). Thus we can choose generators $u, v$ for $M$ such that $u^{a}=u, v^{a}=v^{-1}$. Now let $H$ be the splitting extension of $M$ by $S_{3}$, and note that $M$ is then the unique minimal normal subgroup of $H$.

We now construct two different faithful irreducible modules for $H$ over $G F(3)$, $U_{1}$ and $U_{2}$, such that $U_{1} H$ is $\mathscr{P}^{*}$ and $U_{2} H$ is not $\mathscr{P}^{*}$. Both are induced from modules for $M\langle a\rangle$. Let $V_{1}$ be an irreducible module over $G F(3)$ for $M\langle a\rangle$ with kernel $\langle v, a\rangle$, and $V_{2}$ an irreducible module over $G F(3)$ for $M\langle a\rangle$ with kernel $\langle v\rangle$. It is easy to check that $V_{1}$ and $V_{2}$ have dimension six. Put $U_{1}=V_{1}^{H}$, $U_{2}=V_{2}^{H}$. Then we claim that $V_{1}$ and $V_{2}$ are faithful irreducible modules for $H$. That they are faithful is clear. To see that they are irreducible, observe that if $W$ is a faithful irreducible module for $H$ over $G F(3)$, and $K$ an algebraically closed field of characteristic 3, we have $W^{K}=\sum_{i=1}^{n} W^{i}$, where the $W^{i}$ are all faithful and absolutely irreducible of the same dimension, and $n \geqq 6$ (from [4] 70.15). Also, a athful irreducible module for $H$ over an algebraically closed field has dimension at least 3 (this comes from applying Clifford's Theorem [4] 49.2 to $W_{M\langle b\rangle}^{i}$ and then Blichfeldt's Theorem to an irreducible component of $W_{M\langle b\rangle}^{i}$ ). It follows that $W$ has dimension at least 18 , and then since $H$ has a unique minimal normal subgroup and $U_{1}, U_{2}$ have dimension 18 , that $U_{1}$ and $U_{2}$ are irreducible. 
Using the Mackey subgroup Theorem, we get $U_{1 S_{3}}$ and $U_{2 S_{3}}$ are both direct sums of principal indecomposable modules for $S_{3}$ over $G F(3)$, and that for $U_{1 S_{3}}$ they are all isomorphic to $P_{1}$, and for $U_{2 S_{3}}$ they are all isomorphic to $P_{2}$. Here $P_{1}$ is the principal indecomposable for $S_{3}$ over $G F(3)$ whose socle is the trivial module for $S_{3}$, and $P_{2}$ the principal indecomposable whose socle is a non-trivial module for $S_{3}$.

Now put $G_{1}=U_{1} H$, and $G_{2}=U_{2} H$. Then we claim that $G_{1}$ is a $\mathscr{P} *$ group. Consider them separately.

1) The non-trivial normal subgroups of $G_{1}$ are $U_{1}, U_{1} M, U_{1} M\langle b\rangle$. Clearly for $U_{1}, U_{1} M\langle b\rangle$ any minimal supplement is a complement. So we are left with $U_{1} M$. It is also easy to see that any minimal supplement for $U_{1} M, L$ say, has order prime to 7 . Hence (replacing $L$ by a conjugate if necessary) we may assume that $L$ is a subgroup of $U_{1} S_{3}$, and that $a \in L$. The Sylow 3-subgroup $S$ of $L$ is normal in $L$ and is a subgroup of $U_{1}\langle b\rangle$. Consider $S / \Phi S$ : as an $\langle a\rangle$-module, it is completely reducible, say $S / \Phi S=K_{1} / \Phi S \times \cdots \times K_{n} / \Phi S$. Now not all $K_{i}$ can lie in $U_{1}$ : suppose $K_{1}$ is not contained in $U_{1}$. Then $K_{1}\langle a\rangle$ is a supplement for $U_{1} M$ and hence $L=K_{1}\langle a\rangle$. Thus the Sylow 3-subgroup of $L$ is cyclic, and so $L$ is isomorphic to either $S_{3}$ or $D_{18}$, the dihedral group of order 18. But in $U_{1}\langle b\rangle$, all third powers are acted on trivially by $a$, since third powers lie in the socle of $U_{1 S_{3}}$. Hence $L$ cannot be $D_{18}$, and so $L \cong S_{3}$, giving that $L$ is a complement for $U_{1} M$.

2) We shall find a minimal supplement for $U_{2} M$ in $G_{2}$ which is not a complement. For this, it is enough to show that $P_{2} S_{3}$ contains a subgroup isomorphic to $D_{18}$. To do this, observe that we can choose a basis for $P_{2}, x, y, z$, such that

$$
\begin{array}{ll}
x^{a}=x^{-1} & x^{b}=x y z^{-1} \\
y^{a}=y & y^{b}=y z \\
z^{a}=z^{-1} & z^{b}=z
\end{array}
$$

Now the claim is that $w=b x y^{-1}$ has order 9 and is inverted by $a$ : this is just a matter of checking. We then have $\langle w, a\rangle \cong D_{18}$.

Observe that the subgroup $U_{1} M\langle b\rangle$ of $G_{1}$ is a normal subgroup of nilpotent length three, and has non-abelian Sylow 3 -subgroup, and hence is not a $\mathscr{P}^{*}$ group by Theorem 4.6. The class of $\Phi$-free $\mathscr{P} *$ groups is therefore not normal subgroup closed; cf. 2.8 .

\section{Structure Theorems}

In this section, we shall attemt to shed some light on the structure of Sylow subgroups of $\Phi$-free $\mathscr{P} *$ groups. Although we are mainly interested in $\mathscr{P}^{*}$ groups, 
the crucial lemma of this section is a result about $\mathscr{P}$ groups, and yields some information about the structure of Sylow subgroups in $\Phi$-free $\mathscr{P}$ groups as well as $\Phi$-free $\mathscr{P} *$ groups.

Lemma 4.1. Let $G$ be a $\Phi$-free $\mathscr{P}$ group, $M / N$ a $p$ chief factor of $G, U$ a normal subgroup of $G$, of index $p$, containing the centraliser of $M / N$ in $G$. Then there is an element $y \in G$ with $y$ of order $p^{2}$ and $y \notin U$.

ProOF. This is a fairly standard Hall Higman type argument: we merely sketch it.

Suppose the lemma is false: let $G$ be a minimal counterexample. It is easy to see that $G$ has a unique minimal normal subgroup $M$, with $p$ dividing the order of $M$, that a complement $C$ for $M$ in $G$ acts faithfully and irreducibly on $M$, and that there is a normal subgroup $V$ of $C$ of index $p, U=M V$. If $X$ is a complement for $V$ in $C$, let $X=\langle x\rangle$. Since $C$ is $\Phi$-free $\sigma C=F(C)$, and so $x$ cannot centralise the whole of $\sigma C$ : in particular, there is a minimal normal subgroup $N$ of $C$ such that $[N, x] \neq 1$. Since the order of $N$ is prime to $p$, there is a subgroup $N_{0}$ of $N$ on which $X$ acts faithfully and irreducibly : put $D=N_{0} X$. Consider $M_{D}$. Since $D$ acts faithfully on $M_{D}$, there is a composition factor $W$ on which $D$ acts faithfully and irreducibly. Let $K$ be an algebraically closed field of characteristic $p$. Using [4] 70.15 and Blichfeldt's Theorem, we get thet $W_{X}^{K}$ contains the regular module for $X$, and hence so does $W_{X}$, and then $M_{X}$. But than $M X$ contains an element of order $p^{2}, y$ say. Then $y$ is an element of order $p^{2}$ not in $U=M V$, contradicting the choice of $G$.

COROLlaRY 4.2 Let $G$ be a $\Phi$-free $\mathscr{P}$ group, and suppose that a Sylow psubgroup of $G$ has exponent $p$. Then the Sylow p-subgroups are elementary abelian.

Proof. By induction on the order of $G$. Clearly true if $|G|=1$, so suppose true for all $\Phi$-free $\mathscr{P}$ groups of order less than $|G|$. If either $G$ has a normal subgroup of order prime to $p$ or a normal subgroup of index prime to $p$, invoke 2.2 or 2.8 to obtain the result. If $G$ is abelian there is nothing to prove. Thus $\sigma G$ $=F(G)$ is a $p$-group, and there is a non-central minimal normal subgroup $N$. If $U$ is a maximal normal subgroup of $G$ containing the centraliser in $G$ of $N, U$ has index $p$, and we can apply Lemma 4.1 to deduce that $G$ has elements of order $p^{2}$, a contradiction.

COROllary 4.3 Let $G, U$, be as in the theorem. Then $G$ is not a $\mathscr{P} *$ group.

Proof. Put $Y=\langle y\rangle$. Then $Y$ is a minimal supplement for $U$ which is not a complement.

The next result is our main structural result for $\Phi$-free $\mathscr{P} *$ groups. 
THEOREM 4.4 Let $G$ be a $\Phi$-free $\mathscr{P} *$ group, and $U$ a normal subgroup of $G$ of index $p$. Then the Sylow p-subgroups of $G$ are elementary abelian.

Proof. Suppose the result is false, and let $G$ be a minimal counterexample. The minimality of $G$ gives immediately that $\sigma G$ is a $p$-group, and it then follows from Lemma 4.1 that $G$ has no normal subgroups of index $p$ containing $\sigma G$. Thus $G=\sigma G . U$. Let $V$ be a normal complement for $\sigma G \cap U$ in $\sigma G$ : then $G=U \times V$, and $|V|=p$. Now $U$ cannot have elementary abelian Sylow subgroups, and so by Corollary $4.2, U$ contains an element $x$ of order $p^{2}$. If $V=\langle v\rangle$, hen $\langle v x\rangle$ is a minimal supplement for $U$ which is not a complement, a contradiction.

CoRollary $4.5 \mathscr{P}^{*}$ is not direct product closed.

Proof. Consider the group $G_{1}$ of $\S 3$, and let $C_{3}$ be the cyclic group of order 3 . By Theorem 4.4, $G_{1} \times C_{3}$ is not a $\mathscr{P}^{*}$ group, though $G_{1}$ and $C_{3}$ are.

THEOREM 4.6. Let $G$ be a $\Phi$-free $\mathscr{P}^{*}$ group, and suppose that $G$ has nilpotent length 3: then $G$ has elementary abelian Sylow subgroups.

Proof. Again, suppose that the result is false, and let $G$ be a minimal counterexample, and let $p$ be a prime for which $G$ has a non-abelian Sylow $p$-subgroup. Then $\sigma G=F(G)$ is a $p$-group, and $F(G / \sigma G)=F_{2} / \sigma G$ is a $p^{\prime}$-group. But then $G / F_{2}$ is nilpotent, and since a Sylow $p$-subgroup of $G$ was assumed non-abelian, $p$ divides $|G| F_{2} \mid$, whence $G$ has a normal subgroup of index $p$, contradicting Theorem 4.4 .

\section{Direct Products}

We have seen that the direct product of two $\Phi$-free $\mathscr{P} *$ groups need not be a $\mathscr{P}^{*}$ group. The best we have been able to do in this direction is the following theorem.

THEOREM 5.1. Let $G$ be a $\Phi$-free $\mathscr{P} *$ group, $\pi$ a set of primes such that $G_{\pi} \in \mathscr{E}_{\pi}$, and $H \in \mathscr{E}_{\pi}$. Then $G \times H$ is a $\mathscr{P}^{*}$ group.

Proof. Put $D=G \times H$. We work by induction on the order of $D$. Let $1 \neq N$ be a normal subgroup of $D$, and $S$ a minimal supplement for $N$ in $D$, that is, $N S=D, S \cap N \leqq \Phi S$. Suppose that $N \cap S \neq 1$.

We first show $H \cap N=H \cap S=1$. Observe that $H \cap N$ is normal in $D$, and $S(H \cap N) /(H \cap N)$ is a minimal supplement for $N /(H \cap N)$ in $D /(H \cap N)$. If $H \cap N \neq 1$, we conclude by induction that $S \cap N \leqq H \cap N$. But then $S \cap N$ is a normal $\pi$-subgroup of $S$, and so by 2.1 has a complement $C_{0}$ in $S$. $C_{0}$ is then a complement for $N$ in $D$, giving $S=C_{0}$, contradicting $S \cap N \neq 1$. Hence $H \cap N$ =1. If $H \cap S \neq 1$, then $H \cap S$ is normal in $D$, and, by induction, there is a complement $C_{1} /(S \cap H)$ in $D /(S \cap H)$ for $N(S \cap H) /(S \cap H)$ (with $C_{1} \leqq S$ ). But then $N C_{1}=D$, and 


$$
N \cap C_{1} \leqq(S \cap H) \cap N=1 .
$$

Hence $S=C_{1}$, again a contradiction, and so $S \cap H=1$ also.

Next note that $N \cap G \neq 1$. For, it not, $N$ is a $\pi$-group, and 2.1 gives us a contradiction again. Also, arguing as in the previous paragraph, if $M$ is a minimal normal subgroup of $G, M \leqq N \cap G$, then $S \cap N \leqq M$. Then, using 2.1 again, $S \cap N$ is a $p$-group for some prime $p$ not in $\pi$.

Let $\sigma$ be the projection of $D$ onto $G$. Since $N \sigma S \sigma=G$, and $G \in \mathscr{P P}^{*}$, there is a subgroup $C$ of $S$ such that $C \sigma$ is a complement for $N \sigma$ in $G$ (observe that $\sigma$ is a monomorphism on $S$ and $N$.) Let $B$ be the normal subgroup of $S$ defined by $B \sigma=N \sigma \cap S \sigma$ : then $C$ is a complement for $B$ in $S$. Since $(S \cap N) \sigma=S \cap N$, we have

$$
S \cap N \leqq B \cap N \leqq S \cap N,
$$

giving $B \cap N=S \cap N$. Further, $B \cap N=B_{\pi^{\prime}}$, where $\pi^{\prime}$ is the set of primes not in $\pi$. This comes from the fact that $B_{\pi^{\prime}}=B_{\pi^{\prime}} \sigma \leqq N \sigma$, whence $B_{\pi^{\prime}} \leqq N$, and on the other hand $B \cap N=S \cap N \leqq B_{\pi^{\prime}}$.

Now the usual Frattini argument, with $S$ acting on the conjugates of $B_{0}$, a complement of $S \cap N$ in $B$, gives

$$
S=(S \cap N) N_{S}\left(B_{0}\right) .
$$

But $S \cap N \leqq \Phi S$, and so $S=N_{S}\left(B_{0}\right)$, giving $S=B_{0} C$. Then $S \cap N=1$, a contradiction.

\section{6. $\mathscr{P}^{*}$ groups with non-trivial Frattini subgroup}

Recall that if $G$ is a finite soluble group, $F(G)$ properly contains $\Phi G$, and $F(G) / \Phi G$ is completely reducible as a module for $G$, and also if $G$ is a $\mathscr{P} *$ group with $\Phi G \neq 1, F(G)$ is a $p$-group for some prime $p$. We start by proving

THEOREM 6.1. Let $G$ be a $\mathscr{P}^{*}$ group with $\Phi G \neq 1$. Then $F(G) / \Phi G$ has at most two irreducible components, and if $F(G)$ is abelian, $F(G) / \Phi G$ is irreducible.

Proof. Since $G \in \mathscr{P}^{*}, F(G)$ has a complement, $C$ say, in $G$. Suppose that

$$
F(G) / \Phi G=T_{1} / \Phi G \times T_{2} / \Phi G \times T_{3} / \Phi G
$$

is a decomposition of $F(G) / \Phi G$, with each $T_{i} \neq \Phi G, i=1,2,3$. Then $T_{i}$ is a nonFrattini normal subgroup of $G$, and $T_{j} T_{k} C(i, j, k$ distinct) is a supplement for $T_{i}$, and so contains a complement $X_{i}$ for $T_{i}$ in $G, i=1,2,3$. Since

$$
X_{i} \cong G / T_{i} \cong(G / \Phi G) /\left(T_{i} / \Phi G\right)
$$

it follows that $X_{i} \cap F(G)=U_{i j} \times U_{i k}$, and $X_{i} \cap F(G)$ has a complement $D_{i}$ in $X_{i}$ which normalises $U_{i j}$ and $U_{i k}$, and $T_{l}=U_{i l} \Phi G, l=j, k$. Note also that $U_{i j}, U_{i k}$ are elementary abelian and $D_{i}$ is a complement for $F(G)$ in $G$. 
Consider first the case in which $\Phi G$ is central in $F(G)$. Then each $T_{l}$ is abelian, $\left[U_{i j}, U_{i k}\right]=1$, and hence $\left[T_{j}, T_{k}\right]=1$. It follows that $F(G)$ is abelian. Since each $U_{i j}$ admits some complement (viz. $D_{i}$ ) of $F(G)$ in $G$, it admits $C$, and hence $U_{21} \times U_{12} \times U_{13}$ is normal in $G$,

$$
\left(U_{21} \times U_{12} \times U_{13}\right) C=G
$$

(since it generates $G$ modulo $\Phi G$ ), and

$$
F(G)=U_{21} \times U_{12} \times U_{13} .
$$

But then $F(G)$ is a direct sum of completely reducible modules and so is completely reducible. Thus $\Phi G=1$, a contradiction.

If $\Phi G$ is not central in $F(G)$, put $N=[F(G), \Phi G]$, and consider $G / N$. Since $N<\Phi G$

$$
\Phi(G / N)=\Phi G / N \neq 1, \quad F(G / N)=F(G) / N, \quad G / N \in \mathscr{P} *,
$$

and we are in the same situation as in the last paragraph.

If $F(G)$ is abelian, we assume a decomposition

$$
F(G) / \Phi G=T_{1} / \Phi G \times T_{2} / \Phi G,
$$

and proceed much as above.

For $G \in \mathscr{P} *$ with $\Phi G \neq 1$, we can say a little more about the structure of $F(G)$, mainly for the case $F(G) / \Phi G$ reducible. If $G \in \mathscr{P P}^{*}, \Phi G \neq 1$, and $F(G) / \Phi(G)$ is reducible, we have, as in the proof of Theorem 6.1, that there exist elementary abelian $p$-subgroups of $F(G), U_{1}, U_{2}$, such that

$$
U_{i} \cap \Phi G=U_{1} \cap U_{2}=1,
$$

$U_{i} \Phi G / \Phi G$ is irreducible, $i=1,2$, and

$$
U_{1} U_{2} \Phi G=F(G) .
$$

THEOREM 6.2. With $G$ as above, we may choose the $U_{1}$ and $U_{2}$ to be normalised by the same complement $C$ of $F(G)$ in $G$. Further, $\Phi(F(G))=\Phi G=F(G)^{\prime}$.

PROOF. The proof separates into two cases, depending on whether one or none of $U_{1}, U_{2}$ is a trivial $G / F(G)$-module

i) Suppose that $U_{2}$ is a trivial $G / F(G)$-module. Then $G / \Phi G$ is a $\Phi$-free $\mathscr{P} *$ group, and has a quotient group of order $p$. Hence the Sylow $p$-subgroup of $G / \Phi G$ is elementary abelian (Theorem 4.4 ), and we deduce that $F(G) / \Phi G$ is the Sylow $p$-subgroup of $G / \Phi G$, and hence $F(G)$ is the Sylow $p$-subgroup of $G$. Now, as in the proof of Theorem 6.1, we see that there are complements $C_{1}$ and $C_{2}$ of $F(G)$ in $G$ normalising $U_{1}$ and $U_{2}$ respectively. By the Schur-Zassenhaus Theorem, they are conjugate: say $C_{1}=C_{2}^{g}, g \in G$. Then $U_{1}, U_{2}^{g}$ both admit $C_{1}$, and have the same properties as $U_{1}, U_{2}$. 
For this case, it is clear that $\Phi(F(G))=\Phi G=F(G)^{\prime}$.

ii) Hence we may suppose that both $U_{1}$ and $U_{2}$ are nontrivial. Let $C$ be a complement for $F(G)$ which normalises $U_{1}$ : we will show that we can choose an elementary abelian p-subgroup of $F(G)$ which complements $U_{1} \Phi G$ in $F(G)$, and is normalised by $C$. We proceed by induction on the class of $F(G)$. By 2.6 we have $Z(F(G))=N \leqq \Phi G$. Consider $G / N$ : if the class of $F(G)=2, \Phi(G / N)=1$, and there is a complement $V / N$ for $U_{1} \Phi G / N$ in $F(G) / N$ which is normalised by $C N / N$ : if the class of $F(G)$ is greater than 2, then by the induction hypothesis, there is a complement $V / N$ for $U_{1} \Phi G / N$ in $F(G) / N$ normalised by $C N / N$. Consider $V C$ : this is a supplement for $U_{1} \Phi G$ in $G$, and hence contains a complement, the compelment having the form $U_{2} D$, where $U_{2}$ is a complement for $U_{1} \Phi G$ in $F(G)$, and $D$ is a complement for $F(G)$ in $G, U_{2} \cap D=1$. Now $U_{2} \leqq V$, $N \leqq V,\left[U_{2}, N\right]=1$, and $V=U_{2} N$, giving $V$ abelian. It follows that $U_{2}$ is normalised by $C$, and $U_{1}, U_{2}, C$ satisfy the requirement of the Theorem.

Next observe that $U_{i}\left[U_{1}, U_{2}\right]$ is a non-Frattini normal subgroup, $i=1,2$, and hence $\Phi G \leqq U_{i}\left[U_{1}, U_{2}\right]$ (by 2.3). But

and so we have

$$
U_{1}\left[U_{1}, U_{2}\right] \cap U_{2}\left[U_{1}, U_{2}\right]=\left[U_{1}, U_{2}\right]
$$

$$
\Phi(F(G)) \leqq \Phi G \leqq\left[U_{1}, U_{2}\right] \leqq F(G)^{\prime} \leqq \Phi(F(G)),
$$

and hence $\Phi(F(G))=\Phi G=F(G)^{\prime}$.

If, in the set up of Theorem $6.2, F(G)$ has class $c$, form the $\mathfrak{N}_{c}$-product $F^{*}$ of $U_{1}$ and $U_{2}$, and, letting $C$ act on $F^{*}$ in the natural way, form the split extension $F^{*} C=G^{*}$. Clearly $G$ is a homomorphic image of $G^{*}$, and one might hope that a $G^{*}$ formed with appropriate $U_{1}, U_{2}, C$ is a $\mathscr{P}^{*}$ group. This is not in general true, and it is not difficult to find examples.

As an example, let $V$ be the elementary abelian group of order 4 , acted on non-trivially by $C_{3}$ (the cyclic group of order 3), and $C_{2}$, acted on trivially by $C_{3}$. Let $F$ be the free $\mathfrak{N}_{2}$-product of $V$ and $C_{2}$, and $G=F \cdot C_{3}$ then $\left\langle\Phi F, V, C_{3}\right\rangle=N$ is a normal subgroup of index 2 in $G$ and there are elements of order 4 not in $N$, whence $G$ is not a $\mathscr{P}^{*}$ group. This example is not atypical of the situation when one of $U_{1}$ and $U_{2}$ is trivial: we can show that $F(G)$ cannot be a 2-group, or more generally, if $F(G)$ is a $p$-group of class $p$, it must have exponent $p$. It is for this case that the analogy with Bechtell's results is closest.

Finally, if $F(G) / \Phi G$ is irreducible, we can say nothing more in general: however, we can prove

THEOREM 6.3. Let $G$ be a group with the following properties:

(i) $G / \Phi G$ is a $\mathscr{P} *$ group

(ii) $F(G)$ is a p-group for some prime $p$, and $F(G) / \Phi G$ is irreducible, and

(iii) $(p,|G / F(G)|)=1$.

Then $G$ is a $\mathscr{P}^{*}$ group. 
Proof. From (iii) we deduce $\Phi(F(G))=\Phi G$ : from (ii) that $G / \Phi G$ has a unique minimal normal subgroup $F(G) / \Phi G$.

Let $N$ be a non-Frattini normal subgroup of $G$. Then $N \Phi G \geqq F(G)$, and hence $N \geqq F(G)$. Let $S$ be a minimal supplement for $N$ : then $S \Phi G / \Phi G$ is a minimal supplement for $N / \Phi G$, and since $G / \Phi G$ is a $\mathscr{P *}$ group, $S \cap N \leqq \Phi G$. But then from (iii)

$$
(|S| S \cap N|,| S \cap N \mid)=1,
$$

and so by the Schur Zassenhaus Theorem $S \cap N$ has a complement $C$ in $S$ : and clearly $N C=G$, giving $S=C, S \cap N=1$, i.e. $S$ is a complement for $N$ in $G$.

\section{References}

[1] H. Bechtell, 'A generalization of Hall-complementation in finite supersolvable groups', Trans. Amer. Math. Soc. 140 (1969), 257-270.

[2] C. Christensen, 'Groups with complemented normal subgroups.' J. London Math. Soc. 42 (1967), 208-216.

[3] C. Christensen, Unpublished.

[4] C. W. Curtis, and I. Reiner, Representation theory of finite groups and associative algebras (Interscience, New York (1962)).

[5] W. Gaschütz, 'Zur Erweiterungstheorie der endlichen Gruppen', J. Reine Agnew. Math. 190 (1952), $93-107$.

[6] W. Gaschütz, 'Uber die $\Phi$-Üntergruppe endliche Gruppen', Math. Z. 58 (1953), 160-170.

Pure Mathematics Department

School of General Studies

Australian National University

Box 4, P.O. Canberra, A.C.T.

Australia 